\title{
Museografía interactiva: el pasado y presente de las telecomunicaciones
}

\section{Artículo de reflexión}

\section{Carlos Augusto Bahamón Cardona}

Universidad Militar Nueva Granada (Cajicá, Colombia) carlos.bahamon@unimilitar.edu.co

Recibido: 12 de junio de 2017

Aprobado: 20 de septiembre de 2017

Cómo citar este artículo: Bahamón Cardona, Carlos Augusto. (2018) Museografía interactiva: el pasado y presente de las telecomunicaciones. Calle14: revista de investigación en el campo del arte 13 (23) pp. 122-133. DOI: https://doi.org/10.14483/21450706.12993

Agradecimientos: Vicerrectoría de Investigaciones, Universidad Militar Nueva Granada. Museo Nacional de las Telecomunicaciones; Ingeniera Ada Echávez e Ingeniero Mateo Forero. Facultad de Ingeniería, Campus Nueva Granada 



\title{
Resumen
}

El proyecto de reapertura del Museo Nacional de las Telecomunicaciones (MNT), dirigido por la Universidad Militar Nueva Granada (UMNG), afronta los retos que impone la sociedad colombiana en la actualidad sobre la relación que tiene la museografía con aspectos educativos, culturales y sociales de nuestro país. Es así como la Facultad de Ingeniería de la UMNG se involucra en este proyecto, planteando el desarrollo de ambientes multimediales y de apuestas interactivas que permitan acercar la noción de museo a la comunidad en general y especialmente la universitaria. Se asume a la vez, la misión de trascender el arquetipo tradicional museístico y equiparar la experiencia historiográfica del MNT con las grandes apuestas digitales, hipermediales y desarrollos de estudios de interactividad logrados en los más importantes museos del mundo.

\section{Palabras claves}

Museo, museografía, multimedia, experiencia, interactivo, historiografía, hipermedial, cultura, Colombia.

\section{Interactive museography: the past and present of telecommunications}

\begin{abstract}
The project for the reopening of the National Museum of Telecommunications (MNT), by the Nueva Granada Military University (UMNG), addresses the challenges currently posed by Colombian society regarding the relation of museography to some educational, cultural and social aspects of our country. This is how the UMNG Engineering Faculty engages in this project, proposing the development of multimedia environments and an interactive commitment that fosters the rapprochement of the museum to the community in general and especially the university. At the same time, we take on the mission of transcending the traditional museum archetype and equating the historiographic experience of the MNT with the great digital and hypermedia ventures and with the developments in interactivity studies achieved in the most important museums of the world.
\end{abstract}

\section{Keywords}

Museum, museography, multimedia, experience, interactive, historiography, hypermedia, culture, Colombia.

Muséographie interactive : le passé et le présent des télécommunications

\section{Résumé}

Le projet de réouverture du Musée national des télécommunications (MNT) par I'Université militaire Nueva Granada (UMNG) aborde les défis actuels de la société colombienne concernant la relation entre la muséographie et certains aspects éducatifs, culturels et sociaux de notre pays. C'est ainsi que la Faculté d'Ingénierie UMNG s'engage dans ce projet, proposant le développement d'environnements multimédia et un engagement interactif qui favorise le rapprochement du musée à la communauté en général et surtout à l'université. En même temps, nous assumons la mission de transcender l'archétype traditionnel du musée et d'assimiler l'expérience historiographique du MNT aux grandes entreprises numériques et hypermédia et aux développements des études d'interactivité réalisées dans les musées les plus importants du monde.

\section{Mots clés}

Musée, muséographie, multimédia, expérience, interactif, historiographie, hypermédia, culture, Colombie.-5- Museografia interativa: 


\section{Resumo}

O projeto para a reabertura do Museu Nacional de Telecomunicações (MNT), pela Universidade Militar Nova Granada (UMNG), aborda os desafios atualmente colocados pela sociedade colombiana sobre a relação da museografia com alguns aspectos educacionais, culturais e sociais de nosso país. É assim que a Faculdade de Engenharia da UMNG se envolve neste projeto, propondo o desenvolvimento de ambientes multimídia e um compromisso interativo que promova a aproximação do museu à comunidade em geral e especialmente à universidade. Ao mesmo tempo, assumimos a missão de transcender o arquétipo do museu tradicional e equiparar a experiência historiográfica do MNT com os grandes empreendimentos digitais e hipermídia e com os desenvolvimentos em estudos de interatividade alcançados nos mais importantes museus do mundo.

\section{Palavras-chave}

Museu, museografia, multimídia, experiência, interativo, historiografia, hipermídia, cultura, Colômbia.

Museografiamanda chagpirirka: antiwa i kunaura kagta kai rimanakuikuna Di. Carlos Augusto Bahamón Cardona

\section{Maillallachiska:}

Kaiarir kai ruraikuna chi parlukunamanda (MNT) kaiarirka kai atun wasi suti Universidadmanda militarmanda granadamanda (UMNG), kai chaiami kawanga imasami kakai atun pugluculumbiamanda mandag chasami ka kunaura kai kilkaikuna i iachagchingasina imasami kachi kaugsai Nukanchipa paismanda. Chasami kai ingenieromanda UMNG aidami kai ruraipi i ruraspa kai suma kagpita kai atun ruraikunapi i aidachinakuka Tukui kaskamanda kai universidadmanda. Chasami kaiari kai arquetipomanda chasami kawachiringa sug museokinamanda i chasami parlu tianga imasami rurarkakuna del MNT kai atun tiaskakunamanda suti digitalkunamanda i achaikuna ruraikunawa chasami kai ruraikuna kami Tukui kai atun museokunamandapi.

\section{Rimangapa Ministidukuna:}

Imasami ruraskapi kagta, iachaikungapa museomanda, multimediamanda iachaikuna, interactivomanda, antiwamanda parlukuna, hipermedial, Nukanchipa kaskamanda, kulumbiamanda. 


\section{Introducción}

La constitución del Museo Nacional de la

Telecomunicaciones (MNT), permite hoy la posibilidad de importantes intercambios epistémicos y técnicos entre la academia de estudios superiores y la tecnología, utilizando la lógica interactiva de los medios contemporáneos digitales de interrelación humana, como dispositivo de conocimiento efectivo para divulgar la importancia histórica de las telecomunicaciones en Colombia. Uno de los retos en la configuración del museo actual, plantea la búsqueda de nuevos lenguajes de comunicación que incrementen el interés en los objetos y las relaciones históricas culturales que lo hacen valioso y que justifican su conservación, así como los distintos espacios de valoración reflexiva para pensar las grandes transformaciones sociales a través de las tecnologías.

En esta perspectiva, el diseño y la ingeniería juegan un papel fundamental en el proceso comunicativo, sensorial y contextual para la noción de museo, emergiendo conceptos favorables de interpretación sobre los espacios museográficos. Tienen la tarea abierta de aplicar los conocimientos para la producción y diseño de medios interactivos porque otorgan un valor agregado a la hora de aprender, examinar, estudiar y disfrutar de una exposición.

El MNT, fundado en 1977 por la Empresa Nacional de Telecomunicaciones - TELECOM, se instala en el Campus Cajicá de la Universidad Militar Nueva Granada - UMNG (Universidad Militar Nueva Granada, 2016) para exponer la colección de distintos equipos y elementos históricos encontrados en oficinas, empresas públicas y particulares de todo el país, permitiendo su disposición al beneficio del público en general. Después de haber sido disuelta la empresa TELECOM, las piezas del museo las adquiere la Universidad Militar Nueva Granada con el fin de darle continuidad a la preservación de los objetos y generar un valor trascendental en la historia de las Telecomunicaciones en Colombia. Actualmente, el Museo se encuentra en reactivación y montaje, con el fin de mostrarles a sus visitantes una exhibición didáctica e interactiva.

\section{Materiales y métodos}

Los museos en Colombia hoy atraviesan una etapa de reflexión y replanteamiento constante sobre la forma en que se muestran a sus audiencias, pues los medios tradicionales (televisión, radio e impresos) e incluso los actuales como el internet no son suficientes para suscitar el interés deseado en el espacio museográfico. Se hace necesario entonces, que los museos planteen nuevas estrategias para mejorar su reconocimiento y aceptación en un público cada vez más interesado en otros discursos sociales y culturales, en los que el museo desde su interpretación clásica, ya no es lo suficientemente atractivo. Es por esto, que el museo debe ofrecer alternativas en sintonía con las dinámicas contemporáneas socioculturales, lo cual posibilite un mayor número de visitas y experiencias satisfactorias a todos sus visitantes.

Este artículo, producto del proyecto de investigación ING 1774 "Museografía interactiva: El pasado y presente de las telecomunicaciones", ha servido como marco teórico para otras investigaciones, por ejemplo: el proyecto de iniciación científica PIC 2255 "Efectos visuales aplicados en el diseño museográfico" en donde los alumnos Laura Tatiana Nieto y Juan Pablo Rodríguez exploran de la misma manera los nuevos campos de la interactividad contribuyentes a forjar una experiencia museográfica enriquecedora tomando como objeto de estudio el MNT, su valor histórico para el país y su instalación reciente en el campus universitario de la Universidad Militar Nueva Granada. Así pues, el resultado de valorar los antecedentes de las Telecomunicaciones será la ruta para proponer desarrollos multimediales que permitan la aplicabilidad en una interacción que incentive el conocimiento histórico de sus visitantes.

\section{El valor histórico del MNT}

Los antecedentes históricos de las telecomunicaciones en Colombia se remontan hasta casi el momento mismo de la invención del telégrafo y el teléfono, pioneros de la época, pues desde 1847 el gobierno del general Tomás Cipriano de Mosquera pensó en instalar dichos avances tecnológicos en nuestro territorio, como parte de las reformas de la estructura económica colonial. En aquella época, funcionaba un ineficiente sistema de correos cuyo funcionamiento no había variado desde la época colonial. Las comunicaciones eran muy difíciles pues varias regiones estaban aisladas entre sí, por los trascendentes aspectos geográficos colombianos que afectaban la identidad nacional, como las cordilleras y los numerosos ríos en los que la infraestructura vial y fluvial estaba muy deteriorada.

Predominaban los medios tradicionales y rústicos de comunicación. Ejemplo de ello puede verse aún hoy en la zona selvática amazónica donde las comunidades 


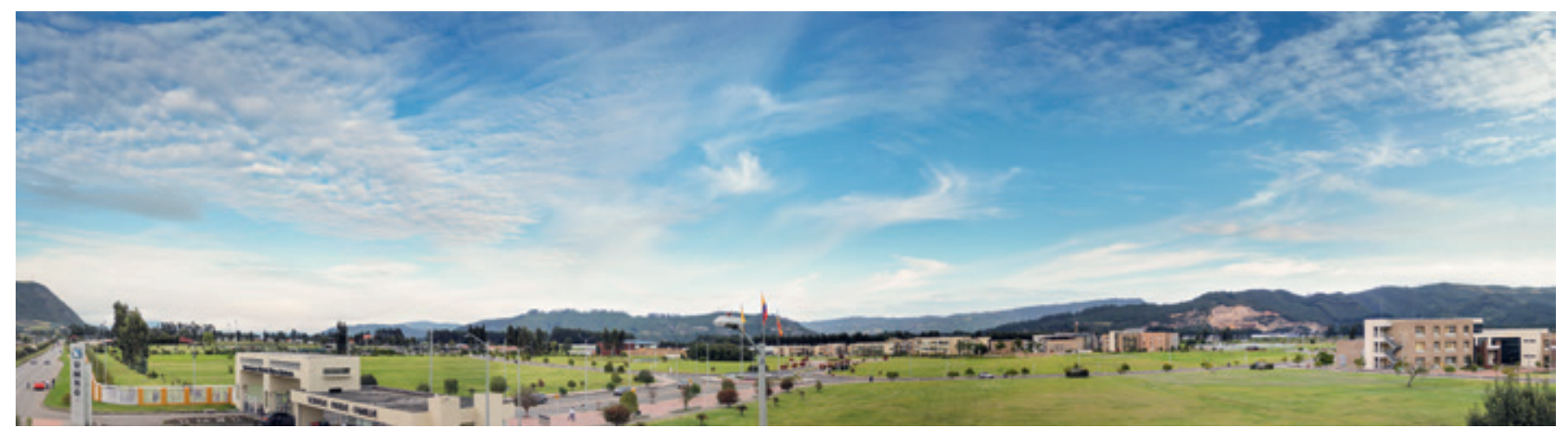

Fig. 2. Campus, Universidad Nueva Granada.

ancestrales o nativas utilizan un sistema de comunicaciones inalámbrico, conocido como maguaré, que consistía en dos tambores golpeados con un mazo de caucho que emitían señales sonoras con un código especial conocido por sus operadores. Estas eran enviadas hasta una distancia de 20 kilómetros, donde eran retransmitidos por otros y con los cuales se comunican acontecimientos importantes de las comunidades, como convocatorias a reuniones tribales, religiosas o festivas (Meyer, Dentel y Seifart, 2012).

El primer telégrafo eléctrico en Colombia, fue instalado por el mismo Samuel Morse ${ }^{1}$. Se inauguró en Panamá, que en ese entonces pertenecía a los Estados Unidos de Colombia, el 12 de agosto de 1855. Sólo diez años después, el 01 de noviembre de 1865 el técnico Guillermo Lee Stiles, discípulo de Morse y administrador de la Compañía del Telégrafo Eléctrico Colombiano, instaló el telégrafo eléctrico en Cuatro Esquinas, hoy municipio de Mosquera y ese día transmitió al jefe de estado Manuel Murillo Toro el primer mensaje telegráfico de la historia nacional. El telégrafo fue el primer dispositivo desarrollado para la comunicación eléctrica a distancia. Fue creado, en 1828, por el inventor norteamericano Harrison Dyar. Este telégrafo, funcionaba por medio de una chispa eléctrica aplicada a un papel químicamente tratado, que permitía dejar un mensaje grabado (Gómez, 2012).

Otro avance que adquiere gran relevancia es el teléfono, creado en 1854 por el italiano Antonio Meucci, que por falta de recursos económicos no logró registrar

1 En 1837 Samuel Morse logra el primer telégrafo de gran aplicación. La comunicación se establecía cuando el primer operador pulsaba un manipulador para transmitir un mensaje codificado en alfabeto morse, este llegaba a su destino a través de una línea física sobre postes o por ondas, en donde un segundo operador lo recibía utilizando audífonos y se encargaba de transcribirlo en papel. su patente y le fue finalmente reconocida al inventor Inglés Alexander Graham Bell. En nuestro país, el servicio telefónico fue inaugurado el 3 de diciembre de 1884, iniciando su servicio con una planta de 500 líneas telefónicas entre Chapinero y Bogotá, ésta fue destruida, años después, en un incendio en el marco de la guerra de los 1.000 días (Universidad Militar Nueva Granada, 2015). El servicio telefónico automático se instauró al finalizar la década de 1920, con el establecimiento de la primera central telefónica automática en la ciudad de Pereira y con la importación de las primeras unidades de teléfonos de disco en el país ${ }^{2}$.

Una mejora sustancial en el aparato telefónico se produjo en 1898 en Oslo. El ingeniero eléctrico danés Valdemar Poulsen logra conectar al fonógrafo un teléfono. A este aparato combinado se le denomina telégrafo o telefonógrafo, es decir, grabadora magnética. El aparato es capaz de registrar magnéticamente conversaciones telefónicas que se pueden oír más tarde por el auricular del aparato. Este invento combinado con los logros de la telegrafía sin hilos crea otro de los medios masivos de comunicación: La radio.

Poco a poco el sistema radial mejora su señal y hacia 1906 ya era posible enviar desde un emisor música y palabras. A comienzos de la década de 1920 hay emisoras regulares de radio en varios países. En 1928, Edwin Howard Armstrong propone el uso de frecuencia modulada - FM, de onda un poco menos larga que el AM. Ya en 1929, el presidente de Colombia Miguel Abadía Méndez inauguró la primera radiodifusora llamada HJN.

2 La cabina telefónica que posee el MNT fue utilizada en Bogotá en los años 30 y 40 . Es un teléfono americano de la marca Stromberg Carlsom en madera con magneto, auricular en baquelita negra, fabricado en los años 30. El primer teléfono inventado por el italiano Antonio Meucci, fue el principal medio de comunicación en el siglo XIX. 
A partir de la década de los treinta, se instalan en varias ciudades las emisoras comerciales, generando a lo largo de los años un afincamiento de la radio en el país, incluso cuando la incursión de la televisión suponía un declive en la radio, esta adquirió más popularidad llegando a varios lugares donde habitualmente no llegaba la televisión (Biblioteca Luis Ángel Arango, 2015).

Cuando la Universidad Militar Nueva Granada adquirió la responsabilidad de administrar y poner en funcionamiento nuevamente el museo, luego de quedar muchos años cerrado después de la subasta pública de TELECOM, la cual le otorgó a Telefónica de España más del cincuenta por ciento del control de su capital accionario y causó privatizar lo que fue una empresa estatal (Caracol Radio, 2006). El MNT se concentra en su reactivación y montaje, con el fin de mostrarle a sus visitantes una exhibición didáctica e interactiva, por medio de la cual, se puede comprender y vivir la historia de las Telecomunicaciones en Colombia. La Universidad Militar Nueva Granada acoge el Museo Nacional de la Telecomunicaciones y ahora éste depende directamente de ella y la universidad de la sociedad colombiana, por consiguiente, el hecho de su dependencia ha de reflejarse directamente en los objetivos que se trace el museo y en su razón de ser, para denotar su importancia en el ámbito económico, social y cultural.
Según el estudio que Koester (1993) realiza en "Interactive Multimedia in American Museums" el fin último de los museos ha ido variando a lo largo de la historia conforme cambian los valores de la sociedad que representa, desde el coleccionismo de objetos como símbolo de conquista cultural hasta su papel como exponentes de riqueza o prestigio económico o cultural (Koester y Wright, 1993). En el siglo XVIII las colecciones comenzaron a hacerse públicas, lo que cambió ligeramente la perspectiva que el museo tenía sobre el visitante.

\section{El aporte socioeconómico del museo}

Los museos se han considerado a lo largo de la historia como un espacio destinado al cultivo cultural de las élites y a su entretenimiento. La visión propia de autores como Luis Fernando Ramírez Celis o Violeta Tavizón Mondragón es diferente. Se piensa que la museografía es una herramienta pedagógica que permite compartir una experiencia para ser aprovechada por los demás (Celis y Mondragón, 2012). Lo que muestra un museo y la manera como lo muestra, es una nueva forma de hacer aprendizaje significativo para aplicarlo en nuestro entorno. Luego de visitar el MNT, se aspira que en la mente del visitante queden estas preguntas: ¿Cómo será el teléfono en un futuro cercano? ¿Cómo afectará mi modus vivendi? ¿Qué debo hacer para ir reduciendo

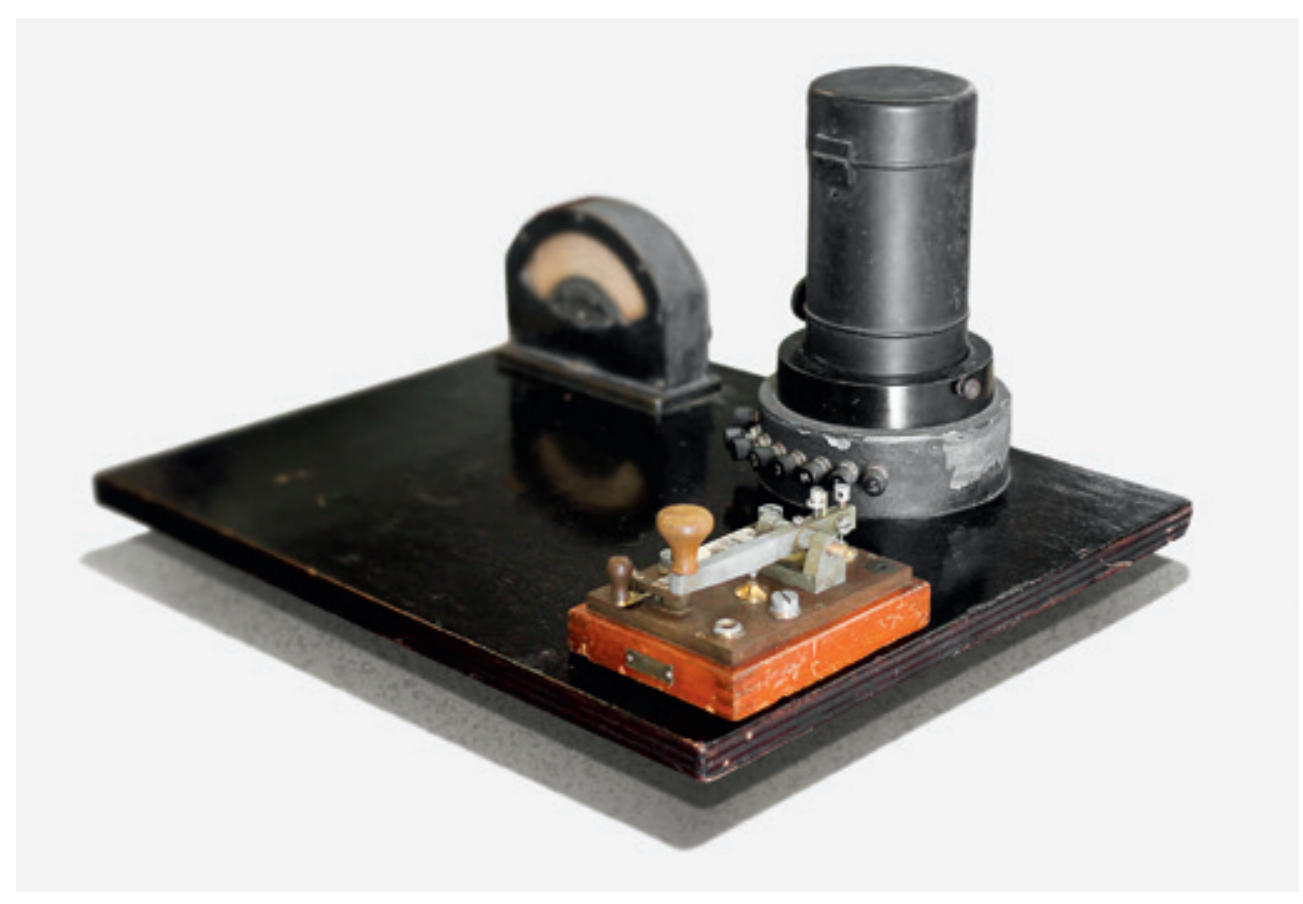

Fig. 3. Telégrafo. 


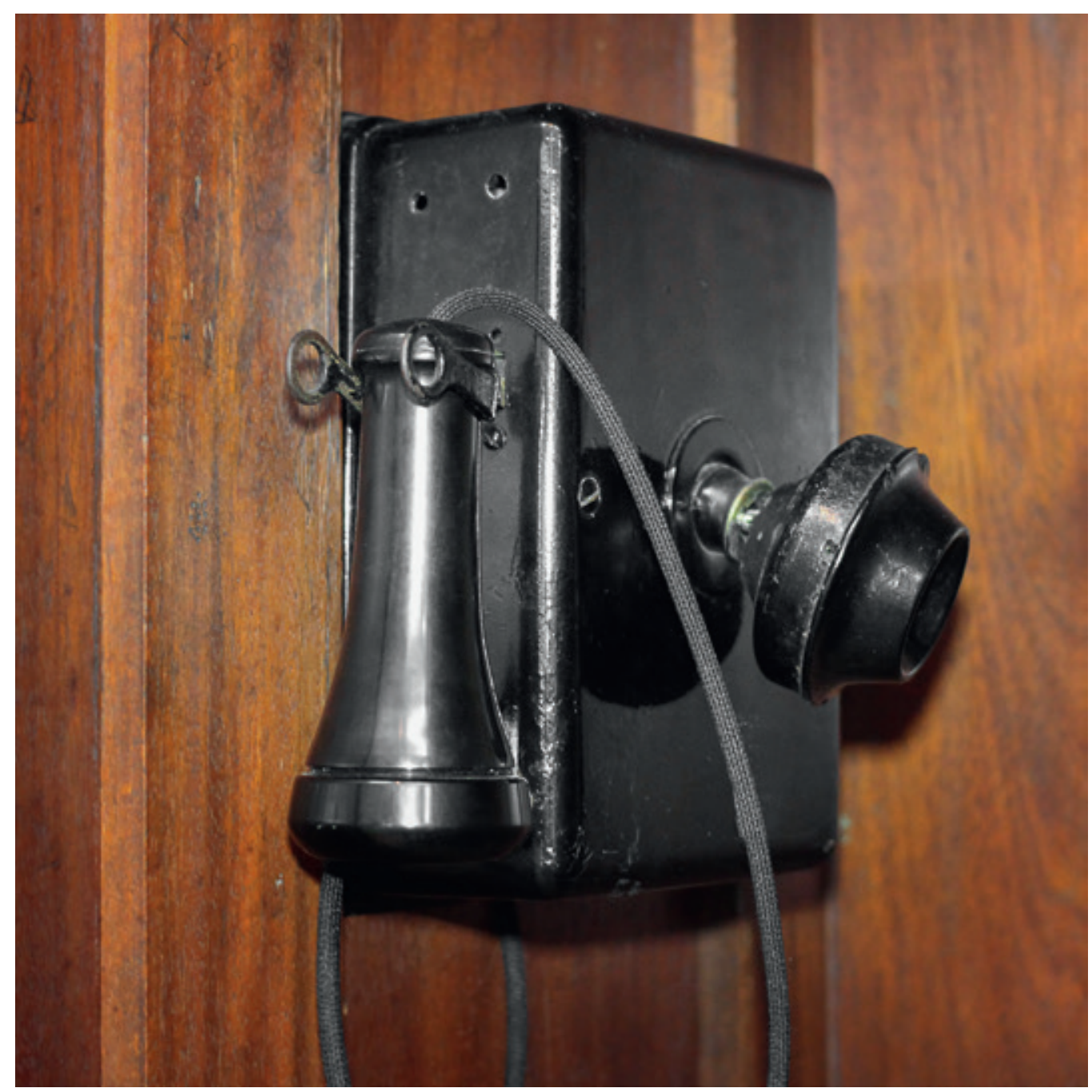

Fig. 4. Teléfono en la abina telefónica utilizada en Bogotá en los años 30 y 40. Fuente: Colección MNT.

el impacto de la brecha tecnológica? y ¿Cómo se combinará y articulará la tecnología de las telecomunicaciones con las demás tecnologías? En otras palabras, los museos generan cuestionamientos desde muchas perspectivas, implicando a todos los que participan de él en una suma de labores, técnicas, estudios y experiencias que compilan las respuestas a los planteamientos de la museografía como herramienta para el aprendizaje, se puede decir entonces que los museos son a la pedagogía y al conocimiento, como las vitrinas a los almacenes.

Se pone por caso, la necesidad de revivir conceptos históricos comparándolos con la actualidad de las comunicaciones, que incluyen muchas tecnologías como la radio, televisión, teléfono y telefonía móvil, bases de datos, redes informáticas o Internet. Gran parte de estas tecnologías, que nacieron para satisfacer necesidades científicas, son expuestas en el museo, razón por la cual la temática del MNT constituye hoy en día una fuente de conocimiento de alto impacto y pertinencia para la sociedad.

\section{Resultados y discusión}

La utilización de herramientas multimedia ha abierto interesantes e impresionantes posibilidades para la comunicación del valor histórico y cultural presente en los museos. Si las tecnologías digitales existen y actualmente apoyan muchos y distintos sectores sociales o industriales ${ }^{3}$; ahora han entrado a los museos para contribuir en una primera instancia con la digitalización de sus colecciones, compartir datos en línea, ofrecer información y recursos que mejoren y optimicen las

3

El sector social e industrial se ve referido a: los medios de comunicación, la medicina, las fuerzas armadas o en el sector del ocio y entretenimiento. 

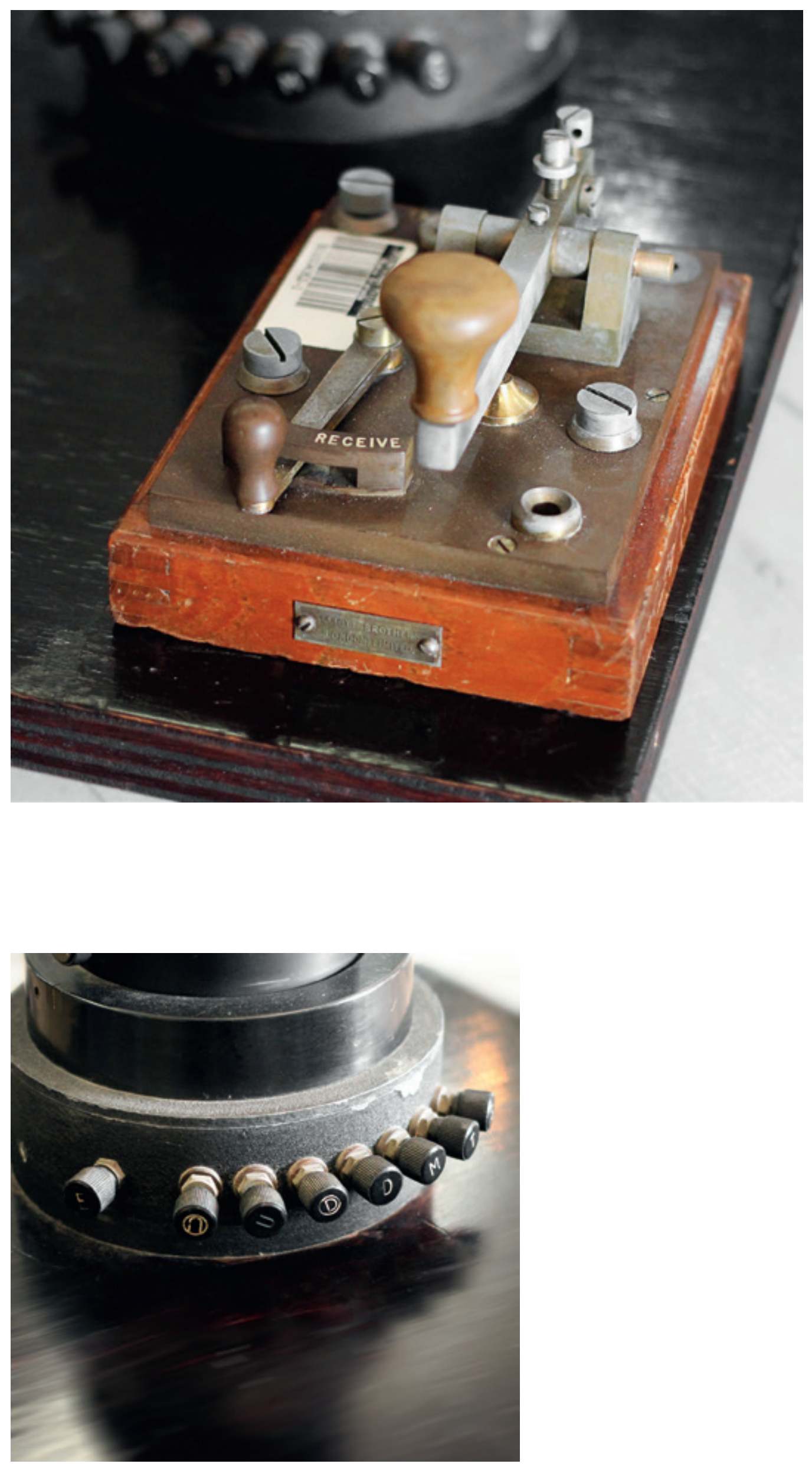

Figs. 5 y 6 . Telégrafo. 
visitas a través de sus páginas web, empleando la tecnología digital como una nueva herramienta de comunicación efectiva con el público que puede ser general o muy específico.

Ahora, se puede decir que el museo que sólo almacena, que es tradicional y común, va a desaparecer si su única intención es la de conservar sus colecciones. Por esta razón el nuevo museo MNT tiene como objetivo principal, reinventar su forma de exponer para divulgar sus contenidos, objetos y colecciones a las visitas presenciales dentro o fuera de sus instalaciones. Así, es que se logra reducir la brecha digital relacionada entre la sociedad y el acceso al conocimiento. Vale la pena retomar en este punto que es necesaria una intensa y continuada labor de digitalización del patrimonio, sobre todo del que pueda estar en peligro de desaparecer y aun de ser olvidado.

En los últimos años muchos museos se han comprometido en proyectos de digitalización. Es posible que esta tarea resulte innecesaria si no se desarrolla siguiendo directrices que permitan una correcta catalogación e integración con las bases de datos virtuales de otros museos o instituciones culturales nacionales e internacionales.

Por otro lado, muchos analistas piensan que la actual Sociedad de la Información (SI) evolucionará, gracias a las TIC, hacia la Sociedad del Conocimiento, en la que la actual gestión documental dé paso a la gestión del saber mediante herramientas desarrolladas para entornos de aprendizaje colaborativo que se alimenten constantemente, esto ya se conoce como la Web 3.0 y la inteligencia artificial (Tuvella y Vilaseca, 2005).

Ahora los museos están inmersos entre conceptos nuevos como la inteligencia artificial, miran hacia nuevas formas de percepción a través de la interacción. Cada vez más museos de arte apuestan por su uso cumpliendo sus funciones educativas, informativas y de difusión. La interacción es pertinente entonces, como herramienta didáctica e informativa de gran utilidad. Al analizar este avance digital y sus innovaciones en los espacios artísticos del mundo, surge la interrogante autocrítica sobre la opción de dejar de digitalizar catálogos y empezar a explorar otras opciones inteligentes que sean efectivas para la catalogación y por consiguiente para la comunicación entre el museo y su público.

En la actualidad una gran cantidad de páginas web de instituciones culturales y museos siguen un modelo

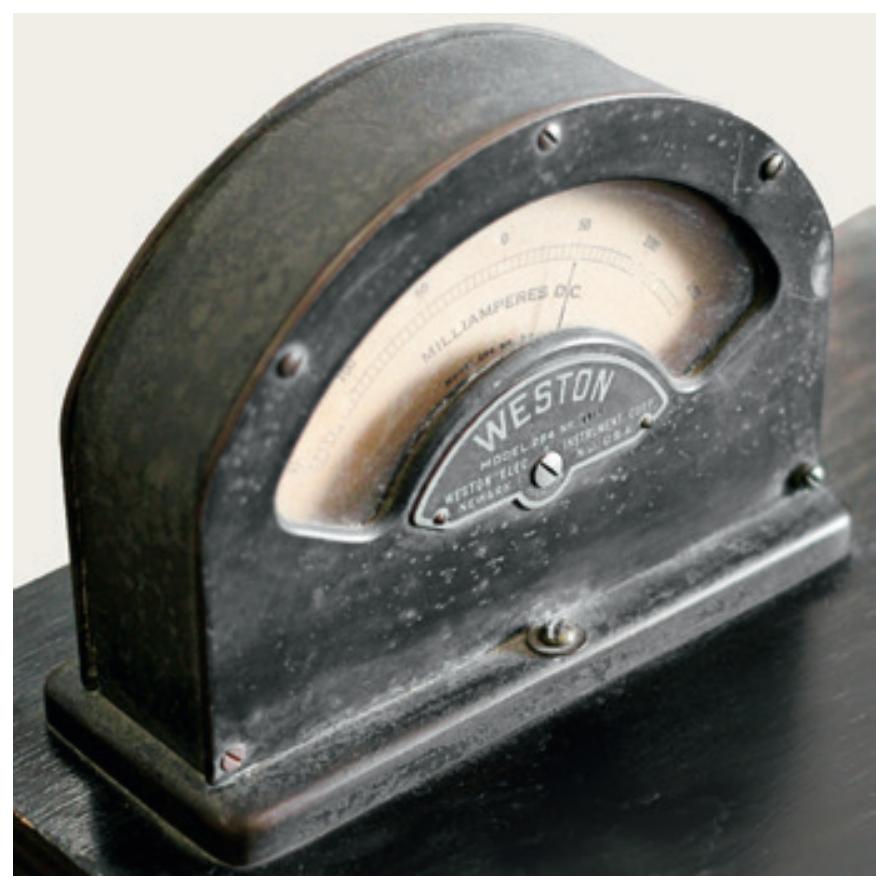

Fig. 7. Telégrafo.

simple básicamente informativo. No obstante, hay otras que son diseñadas para ofrecer contenidos que complementen la visita física antes, durante y después de la misma. En un mundo globalizado, donde el conocimiento científico y el desarrollo tecnológico son primordiales como motores de desarrollo, es necesario impulsar las Tecnologías de Información y Comunicación - TIC, gracias a herramientas con que se dispone actualmente, es posible desarrollar diferentes espacios interactivos multimedia como por ejemplo: visitas virtuales, mapas, juegos, aplicaciones de realidad aumentada, unidades didácticas para profesores, infografías animadas, etc. que hacen posible esta experiencia y contribuyen a un mejor posicionamiento en Internet.

\section{El museo del siglo XXI se abre a todos}

Actualmente existen museos en diversos temas, en todas partes del mundo, desde el Museo de la Ciencia de Londres, el Centro Espacial de Houston, hasta al Centro Interactivo Maloka reforzaron sus planteamientos didácticos con una metodología de interactividad, que apuesta por hacer participar al visitante en el proceso de instrucción a partir de acciones de manipulación directa, "interacción", desarrollo de procedimientos o resolución de problemas. Estos museos evidenciaron la necesidad de aumentar los elementos de intermediación entre el visitante y la colección, y que esta comunicación interactiva no solo ayudaba a potenciar el factor 
didáctico, sino que también, podía lograr mayor eficacia educativa en el visitante, porque se convertía en el protagonista de la investigación al exigirle interactividad: acción y diálogo proactivo con los objetos expuestos.

También son interesantes las recreaciones virtuales de espacios que incorporan información para a contextualizar el activo patrimonial. La visita virtual a la Alhambra de Granada, incluida en el portal web ArsVirtua.com, así como otros grandes monumentos o construcciones del mundo y desarrollada por Fundación Telefónica desde el año 2000, es un claro ejemplo de este tipo de soluciones ${ }^{4}$. (Morgan, 2015) (Fundación Telefónica, 2015).

Otro ejemplo, se encuentra en el proyecto Art Project, en el cual Google (2015) ha colaborado con diecisiete museos de todo el mundo. Además de permitir una visita virtual por el interior de una serie de salas escogidas, es posible admirar más de mil obras de los artistas más renombrados de la historia (Google, 2015).

Asimismo, han aparecido innumerables iniciativas artísticas y culturales que tienen en la Red su única localización. Artistas y creadores, a través de los nuevos medios digitales, dan a conocer sus obras sin necesidad de intermediarios. Incluso, están surgiendo museos virtuales que adoptan como comisarios a los propios visitantes, convirtiéndose estos en críticos y generadores del contenido. En estos entornos, el creador se convierte, con el paso del tiempo, en el transmisor de los contenidos que generan otros, dando lugar a ejercicios de conocimiento colectivo.

\section{Conclusiones}

Como se ha comentado, gracias a la digitalización del patrimonio y a las TIC, las instituciones culturales y educativas tienen en su mano la posibilidad de desarrollar contenidos multimedia que permiten divulgar colecciones, exposiciones y actividades. Su producción y distribución debe apoyarse en entornos digitales e interactivos multiplataforma como ordenadores, tabletas, televisión interactiva, smartphones, marketing

\footnotetext{
$4 \quad$ La visita virtual a la Alhambra de Granada y las construcciones y desarrollos por parte de la Fundación Telefónica, son un claro ejemplo en implementación de información contextual por el uso de tecnologías inmersivas, tales como, recorridos virtuales o la fotografía panorámica 360, el uso de impresión en 3D para la recreación de objetos y la implementación del contenido de redes sociales para estimular la participación del público más allá del presencial.
}

dinámico, etc. para facilitar el acceso a la información y el conocimiento a todos los ciudadanos.

Además, como para la mayoría de los ciudadanos, los museos representan un lugar de esparcimiento y diversión. Los museos han tratado de adaptarse a estas nuevas condiciones creando salas más interactivas, en donde el visitante pueda sentirse parte de la exposición, en el cual principalmente se han utilizado las tecnologías como medio para transmitir todos los conocimientos que se pueden encontrar. Pero tal implementación será eficiente como se espera y logrará aumentar el número de visitas presenciales o virtuales, si el museo propone participación y complicidad, y por tanto interactividad. Así pues, la museografía interactiva se vincula plenamente desde este punto de vista al objetivo real de un museo y la comunicación que ofrece al público.

Es así como en este artículo se presentan algunos conceptos y sugerencias para analizar y evaluar las necesidades que el usuario tiene actualmente frente a los museos, así como la aplicación de la tecnología como herramienta fundamental en el diseño museográfico. Aunque parcialmente basado en experiencias en los museos de la DIBAM (Dirección de Bibliotecas Archivos y Museos de Chile), los planteamientos de este artículo se aplican a cualquier museo. (DIBAM, s.f.).

De momento, y antes de que puedan hacerse realidad estas nuevas propuestas museográficas, es importante que museos e instituciones culturales puedan llevar a cabo la urgente labor de conocer y digitalizar los activos que atesoran, para evidenciar y difundir el valor histórico cultural que tiene toda colección museística en cuanto es una muestra abierta de la evolución técnica y sobre todo conceptual del ser humano, de las sociedades y las genealogías culturales que construyen nociones de identidad. Las TIC en este contexto son entonces aquella catapulta que permite revitalizar el interés en la reflexión constante del ser, colocando como medio o canal el espacio museo, en el caso del MNT, considerado como un objeto de estudio para poner en práctica estas nuevas propuestas, en un espacio interactivo contemporáneo inmerso en un campus universitario. Este artículo es entonces, una invitación para compartir con personas de diferentes niveles culturales y estratos sociales los conocimientos y las tecnologías que hemos aprovechado de manera que pueda hablarse de una museografía interactiva. 


\section{Referencias}

Biblioteca Luis Ángel Arango. (25 de agosto de 2015). Biblioteca Luis Ángel Arango. Obtenido de Banco de la República - Subgerencia Cultural: http://www.banrepcultural.org/blaavirtual/ayudadetareas/comunicacion/ la_radio_en_colombia

Caracol Radio (2006). Caracol Radio. Recuperado el 17 de Mayo de 2017, de La venta de Telecom a Telefónica fue un buen negocio para Colombia dice la Contraloría: http://caracol.com.co/radio/2006/04/07/economia/1144415460_273615.html

Celis, L. F. (2012). Museología, curaduría, gestión y museografía. Recuperado el 17 de Mayo de 2017, de http://www.museoscolombianos.gov.co/fortalecimiento/ comunicaciones/publicaciones/Documents/manual_ artes_visuales_mincultura.pdf

Costa, J. (2010). ADGFAD. Recuperado el 2 de 10 de 2015, de http://www.fad.cat/adg/blog/2010/02/09/ tabletas-ipad-de-5300-anos-de-edad/

DIBAM. (s.f.). Dirección de Bibliotecas, Archivos y Museos. Recuperado el 15 de enero de 2016, de http://www.dibam.cl/614/w3-channel.html

Fundación Telefónica (2015). Fundación Telefónica. Obtenido de http://www.fundaciontelefonica.com/ conocenos/memorias/memoria2009/arteytecnologia/ arsvirtual.htm

Gómez, J. C. (2012). La telegrafía: Una revolución en las Telecomunicaciones de Colombia: 1865-1923. Obtenido de Revista Credencial: http://www.revistacredencial. $\mathrm{com} /$ credencial/historia/temas/la-telegrafia-una-revolucion-en-las-telecomunicaciones-de-colombia-1865-1923

Google. (25 de agosto de 2015). Art Proyect. Obtenido de https://www.google.com/culturalinstitute/project/ art-project

Koester, S., y B. Wright (1993). Interactive Multimedia in American Museums. Pittsburgh: Archives \& Museum Informatics.

Meyer, J., Dentel, L., y F. Seifart (2012). A Methodology for the Study of Rhythm in Drummed Forms of Languages Application to Bora Manguaré of Amazon.
Annual Conference of the International Speech Communication Association, 13. (págs. 686-690.). Portland. Center for Spoken Language Understanding.

Morgan, J. (2015). T.J. Foundation. Obtenido de J. Bruneau \& CADRE Laboratory for New Media: http:// arsvirtua.com.

Tuvella Casadevall, I., y J- Vilaseca Requena (2005). Sociedad Del Conocimiento: Como Cambia El Mundo Ante Nuestros Ojos. Barcelona: Fundació per a la Universitat Oberta de Catalunya.

Universidad Militar Nueva Granada (2015). Museo Nacional de Telecomunicaciones. Obtenido de Universidad Militar Nueva Granada: http://www. museosumng.com/tmtc.html

Universidad Militar Nueva Granada (2016). Museo Nacional de las Telecomunicaciones. Obtenido de Universidad Militar Nueva Granada: http://www. museosumng.com/el\%20museo.html

Mondragón, V. T. (2012). Gaceta de Museos. Recuperado el 17 de mayo de 2017, de Narradores de objetos: El curador como mediador entre la obra y el espectador: http://www.ilam.org/viejo/ILAMDOC/gacetademuseos/ Gaceta52.pdf 